\title{
Specialized Orthonormal Frames and Embedding ${ }^{\star}$
}

\author{
Frank B. ESTABROOK
}

Jet Propulsion Laboratory, California Institute of Technology, 4800 Oak Grove Drive, Pasadena, CA 91109 USA

E-mail: frank.b.estabrook@jpl.nasa.gov

Received October 09, 2012, in final form February 12, 2013; Published online February 15, 2013

http://dx.doi.org/10.3842/SIGMA.2013.012

\begin{abstract}
We discuss some specializations of the frames of flat orthonormal frame bundles over geometries of indefinite signature, and the resulting symmetries of families of embedded Riemannian or pseudo-Riemannian geometries. The specializations are closed sets of linear constraints on the connection 1-forms of the framing. The embeddings can be isometric, as in minimal surfaces or Regge-Teitelboim gravity, or torsion-free, as in Einstein vacuum gravity. Involutive exterior differential systems are given, and their Cartan character tables calculated to express the well-posedness of the underlying partial differential embedding and specialization equations.
\end{abstract}

Key words: embedding; orthonormal frames; Cartan theory

2010 Mathematics Subject Classification: 83C20; 57R40; 58A15

\section{Introduction}

The class $C$ of a Riemannian space is the minimum number of extra dimensions required for it to be locally embeddable in a flat space (various signatures possible, which we ignore for the moment; often we write repeated indices as "covariant", but proper signs must be inserted in such summations). Also our discussions are all local, with no global existence implied for solutions of the equivalent partial differential equation. So class $C$ 4-metrics are those embeddable in $4+C$ flat dimensions, $C \leq 6$, and the embedding of their associated orthonormal frame bundles (Cartan moving frame formalism, basis forms $\theta_{i}$, connection forms $\omega_{i j}=-\omega_{j i}, i=1, \ldots, 4$ ) brings in $C+4 C+C(C-1) / 2$ additional orthonormal basis forms $\theta_{A}$ and connection forms $\omega_{i A}=-\omega_{A i}$ and $\omega_{A B}=-\omega_{B A}, A=1, \ldots, C$. Taking $\mu, \nu$, etc. to span the combined range of indices $1, \ldots, 4+C$ of the embedding space, the 1-forms $\theta_{\mu}$ and $\omega_{\mu \nu}=-\omega_{\nu \mu}$ satisfy the Cartan-Maurer structure equations of a flat orthonormal frame bundle $\mathrm{ASO}(4+C) .{ }^{1}$

Isometric embedding requires the $\theta_{A}$ to vanish upon pullback, and together with their exterior derivatives the 2-forms $\omega_{A i} \wedge \theta_{i}$, and dynamic 4-forms derived from the Hilbert Lagrangian, $\omega_{i A} \wedge R_{j k} \wedge \theta_{l} \epsilon^{i j k l}$, they generate well posed exterior differential systems (EDS) $\left(R_{i j}\right.$ are the induced Riemann 2-forms $\left.d \omega_{i j}+\omega_{i k} \wedge \omega_{j}^{k}\right)$ [4]. These isometric EDS's for solutions of ReggeTeitelboim (RT) gravity, of various classes, have been expounded in a recent paper [2].

${ }^{\star}$ This paper is a contribution to the Special Issue "Symmetries of Differential Equations: Frames, Invariants and Applications". The full collection is available at http://www.emis.de/journals/SIGMA/SDE2012.html

${ }^{1}$ Thus Einstein vacuum general relativity can be set as an exterior differential system on the group space ASO(10), of dimension 55. This is arguably an elegant improvement over the more customary setting of this field theory in terms of coordinate components, which means for a first order set of PDE's setting the EDS on the second frame bundle over a four dimensional base, of dimensionality $4+16+40=60$. The first EDS has 21 Cauchy characteristic vectors so in principle 21 variables can be eliminated, leaving a system of partial differential equations in 34 variables, 4 in involution, independent, and 30 dependent. The second setting, when using orthonormal tetrad frames, as in the dyadic and Newman-Penrose formalisms, only reduces to 44 variables but has 10 degrees of gauge freedom together with 10 conservation laws [1]. 
Among the solutions of RT theory are those that additionally annul the compatible Ricci 3 -forms $\omega_{i A} \wedge \omega_{A j} \wedge \theta_{k} \epsilon^{i j k l}$. This more restricted vacuum Einstein theory can conveniently be directly set as a (not strictly isometric) embedding EDS on the orthonormal frame bundle over 10 space, requiring only the four torsion 2 -forms $\omega_{i A} \wedge \theta_{A}$ to pull back to vanish, together with their exterior derivatives and the dynamic Ricci 3-forms. A number of vacuum solutions, as well as other physically interesting non-vacuum metrics, have been classified both by embedding class and motion group, or metric symmetry [6]. Here we treat both EDS's for embedded families of solutions of RT theory with special symmetry and class $\leq 6$, and new EDS for families of solutions of Einstein theory with special symmetry in class 6 .

In [2] an explicit six dimensional framing for embedding static spherically symmetric (SSS) metrics was found, and solutions of Regge-Teitelboim theory reduced to quadrature, using a coordinate imbedding due to Ikeda (other types of class 2 SSS coordinate embeddings exist; with two new ones, all possible have since been given by Paston and Sheykin [5]). The Schwarzschild vacuum solution of orthodox general relativity was a particular one of these. The connection forms there derived were not explicitly given; we write them below in Section 2. We extract the algebraic specialization of the $\mathrm{O}(6)$ frame fibers they imply. The specialization is a set of self closed linear relations between the $\omega_{\mu \nu}$. A closely related specialization is also presented and Cartan characters of embedding RT EDS's using these specialized frames are calculated, showing only the second of them to be well posed. We take it to be the prototype for a new method of frame specialization that can be used for formulating EDS's for families of solutions having specialized metric symmetries.

In Section 3 we outline this technique for specializing the fibers of $\mathrm{ASO}(4+C)$ frame bundles, and present calculated Cartan character tables of EDS's for isometric embedding of RT theory in them, showing them to be well posed and involutory. Section 4 briefly expounds an illustrative toy problem, isometrically embedding a two dimensional variational problem in a frame specialized flat 3-space. In Section 5 we give results for four specializations of the fibers of $\operatorname{ASO}(6,4)$, the orthonormal frame bundle over flat ten-dimensional space. With torsion 2-forms and Ricci 3-forms, we find EDS's for families of Einstein vacuum solutions that are well posed and involutory.

To summarize, we have calculated the Cartan characters, and well posedness, of a number of EDS for embedded 4-spaces in specialized higher dimension flat orthonormal frame bundles, and we conjecture that their solutions have intrinsic symmetry properties such as Riemannian submersions, isometries and Killing tensors. The specializations we consider are sets of self closed linear relations between the $\omega_{\mu \nu}$. Families of metrics with symmetries have heretofore been difficult to treat with Cartan moving frame or tetrad formalism [3].

\section{$2 \quad$ A static spherically symmetric embedding}

In [2], treating class 2 static spherically symmetrical spacetimes, which all have four isometries, they were imbedded in a flat 6 -space coordinatized by $r, \theta, \phi, t, z_{5}, z_{6}$, signature $(-1,-1,-1,1,1,-1)$; The 6 -space was framed with a family of orthonormal hexad bases adapted to the (already known) metrics of the embedded space-times: This framing could have been even more general, and its connection forms were not there written out, but they allow us to induce the specialization of connection forms that succinctly expresses the desired symmetry; the framing is

$$
\begin{aligned}
\theta_{1}= & \left(d r+\left(d z^{5}+d z^{6}\right) c^{\prime}[r]-2 c^{\prime}[r] t\left(2 c^{\prime}[r] t d r+2 c[r] d t\right)\right. \\
& \left.-\left(d z^{5}-d z^{6}\right)\left(g^{\prime}[r]+c^{\prime}[r] t^{2}\right)\right) / \sqrt{1+4 c^{\prime}[r] g^{\prime}[r]}, \\
\theta_{2}= & r d \theta, \quad \theta_{3}=r \sin [\theta] d \phi, \quad \theta_{4}=t d z^{5}-t d z^{6}+2 c[r] d t+2 c^{\prime}[r] t d r,
\end{aligned}
$$




$$
\begin{aligned}
\theta_{5}= & \left(d r+d z^{5}\left(g^{\prime}[r]-c^{\prime}[r] t^{2}+c^{\prime}[r]+1 /\left(2 c^{\prime}[r]\right)\right)-2 t c^{\prime}[r]\left(2 c^{\prime}[r] t d r+2 c[r] d t\right)\right. \\
& \left.-d z^{6}\left(g^{\prime}[r]-c^{\prime}[r] t^{2}-c^{\prime}[r]+1 /\left(2 c^{\prime}[r]\right)\right)\right) / \sqrt{1+4 c^{\prime}[r] g^{\prime}[r]} \\
\theta_{6}= & d r+d z^{5} /\left(2 c^{\prime}[r]\right)-d z^{6} /\left(2 c^{\prime}[r]\right),
\end{aligned}
$$

$c[r]$ and $g^{\prime}[r]$ are arbitrary functions. The flat metric is

$$
-\theta_{1} \theta_{1}-\theta_{2} \theta_{2}-\theta_{3} \theta_{3}+\theta_{4} \theta_{4}+\theta_{5} \theta_{5}-\theta_{6} \theta_{6}
$$

and the embedding is isometric, $\theta_{5}=0, \theta_{6}=0$.

The exterior derivatives of the above, expanded on them, give an explicit set of specialized the 15 connection 1 -forms $\omega_{\mu \nu}$

$$
\begin{aligned}
\omega_{12}= & \frac{\theta_{2}}{r \sqrt{1+4 c^{\prime}[r] g^{\prime}[r]}}, \quad \omega_{13}=\frac{\theta_{3}}{r \sqrt{1+4 c^{\prime}[r] g^{\prime}[r]}}, \quad \omega_{14}=\frac{c^{\prime}[r]\left(\theta_{4}-2 t \theta_{6} c^{\prime}[r]\right)}{c[r] \sqrt{1+4 c^{\prime}[r] g^{\prime}[r]}}, \\
\omega_{15}= & \left(\theta_{1}-\theta_{5}+\theta_{6} \sqrt{1+4 c^{\prime}[r] g^{\prime}[r]}\right)\left(-\left(1+2 c^{\prime}[r] g^{\prime}[r]\right) c^{\prime \prime}[r]\right. \\
& \left.+2 c^{\prime}[r]^{2} g^{\prime \prime}[r]\right) /\left(c^{\prime}[r]\left(1+4 c^{\prime}[r] g^{\prime}[r]\right)^{3 / 2}\right), \\
\omega_{16}= & -\left(\left(\theta_{1}-\theta_{5}\right) \sqrt{1+4 c^{\prime}[r] g^{\prime}[r]}+\theta_{6}\left(1+4 c^{\prime}[r] g^{\prime}[r]\right)\right) c^{\prime \prime}[r] /\left(c^{\prime}[r]\left(1+4 c^{\prime}[r] g^{\prime}[r]\right)^{3 / 2}\right), \\
\omega_{23}= & \frac{\operatorname{Cot}[\theta] \theta_{3}}{r}, \quad \omega_{24}=0, \quad \omega_{25}=-\frac{\theta_{2}}{r \sqrt{1+4 c^{\prime}[r] g^{\prime}[r]}}, \quad \omega_{26}=-\frac{\theta_{2}}{r}, \quad \omega_{34}=0, \\
\omega_{35}= & -\frac{\theta_{3}}{r \sqrt{1+4 c^{\prime}[r] g^{\prime}[r]}}, \quad \omega_{36}=-\frac{\theta_{3}}{r}, \quad \omega_{45}=\frac{c^{\prime}[r]\left(-\theta_{4}+2 t \theta_{6} c^{\prime}[r]\right)}{c[r] \sqrt{1+4 c^{\prime}[r] g^{\prime}[r]}}, \quad \omega_{46}=0, \\
\omega_{56}= & -\left(\left(\theta_{1}-\theta_{5}\right) \sqrt{1+4 c^{\prime}[r] g^{\prime}[r]}+\theta_{6}\left(1+4 c^{\prime}[r] g^{\prime}[r]\right)\right) c^{\prime \prime}[r] /\left(c^{\prime}[r]\left(1+4 c^{\prime}[r] g^{\prime}[r]\right)^{3 / 2}\right) .
\end{aligned}
$$

To generalize from this coordinate presentation, we note from it that the algebraic frame fiber specialization for this SSS embedding is apparently generated by the annulling of seven 1-forms $\omega_{12}+\omega_{25}, \omega_{13}+\omega_{35}, \omega_{14}+\omega_{45}, \omega_{16}+\omega_{65}, \omega_{24}, \omega_{34}, \omega_{64}$. It can be checked that: exterior derivatives of these connection 1-forms all vanish by virtue of the 1-forms themselves. Otherwise said, they are self-closed, or a completely integral Pfaffian system (c.i.p.s.). Let us call them a set of symmetry forms.

We might consider an isometric embedding EDS with this frame specialization, set on the 21 dimensional orthonormal frame bundle over six dimensions $\theta_{i}, i=1, \ldots, 4$ and $\theta_{A}, A=5,6$. The EDS is generated by the nine 1-forms $\left(\theta_{A}\right.$, symmetry forms), together with their closure 2 -forms. Taking the signature $-1,-1,-1,1,1,-1$ we calculate the EDS to have Cartan character table $21(9,2,2,2) 4$ and to be in involution with respect to the $\theta_{i}$. Since $\omega_{23}$ does not appear in the generators there is one Cauchy Characteristic vector. There is one degree of gauge freedom: $s_{4}=1$. If we further add to this EDS the two dynamic 4-forms of Regge-Teitelboim gravity, however, we no longer have a well posed system: all four $\theta_{i}$ are then not in involution. So this isometric embedding EDS is apparently not exactly equivalent to the coordinate presentation of [2]. The coordinate presentation has of course further specialized the framing (only two arbitrary functions of one metric variable come in), and we are in this present work concerned with finding well posed involutory families of symmetric solutions.

A possible resolution discussed in Section 3 is to consider an EDS generated by the closely related c.i.p.s. $\omega_{12}+\omega_{25}, \omega_{13}+\omega_{35}, \omega_{14}+\omega_{45}, \omega_{16}+\omega_{65}, \omega_{42}+\omega_{26}, \omega_{43}+\omega_{36}$. This set of six symmetry forms leads to a well posed class 2 EDS for RT theory, with character table $21(8,2,2,4) 4$, in involution with respect to the $\theta_{i}$ and has one degree of gauge freedom $s_{4}=1$. 


\section{A systematic specialization of frames in embedding spaces}

The first four of the symmetry forms for Schwarzschild embedding found above are a c.i.p.s. in their own right: $\omega_{12}+\omega_{25}, \omega_{13}+\omega_{35}, \omega_{14}+\omega_{45}, \omega_{16}+\omega_{65}$. The exterior derivative of any of these pulls back to vanish modulo the set. This is also true if all four are written with minus signs between the two terms. The index 1 , or in general $i$, is from the set of imbedded forms, and the index 5 or in general $A$ is from the coset of embedding dimensions. The pulled back form $\omega_{15}$, or in general $\omega_{i A}$, is exact modulo the set. Remarkably, this construction of a c.i.p.s. works for all dimensions and signatures, so long as the signatures of $i$ and $A$ are of opposite sign! We remember the Codazzi equation $d \omega_{i A}+\omega_{i \mu} \wedge \omega_{\mu A}=0$ and notice that all the quadratic terms vanish modulo these c.i.p.s. This is the most basic construction of specializing symmetry forms we have found.

Further systematic addition of these specializing sets of symmetry forms can go on as long as more pairs, say $j, B$, etc., of opposite signature are available (omitting previous $i$, $A$, etc. pairs from each successive summation), and a number of Cartan characteristic table calculations have shown that well posed RT (isometric) EDS's are always found. Moreover, we find this again in the class 6 case for Einstein vacuum (torsion-free embedding). In Section 5 we explicitly write these four c.i.p.s., having numbers of generators $8,8+6=14,8+6+4=18$ and $8+6+4+2=20$ respectively.

Our systematic construction of these c.i.p.s. for specializing frame curvature forms, in $\operatorname{ASO}(n, m)$, appears to be new, but a number of other c.i.p.s. for specialized frames are of course well known and important. When a c.i.p.s. is imposed on the fiber algebra $\mathrm{O}(n, m)$ the remaining moving frame forms are those of a subbundle, which can be labeled by its fiber algebra and base dimension. For example the case of a subbundle $\operatorname{ASU}(m)$ of $\operatorname{ASO}(m, m)$, and further isometric embedding of $\mathrm{ASO}(m)$ in that, is explicitly treated as an EDS for special Lagrangian calibration in [4, p. 201] (the Cartan character table given there should read $s_{2}=n-1$ ). Various other interesting manifolds discussed there similarly can be understood as discovery of c.i.p.s. for subbundles of orthonormal frame bundles $\operatorname{ASO}(m, n)$; taken together with isometric embedding derived from minimizing calibration forms, EDS are found for associative, coassociative and Cayley geometries. We should also stress that, in distinction to previous such work (e.g., Calabi-Yau manifolds), our new specializations have so far only been been treated locally, through calculation of Cartan characters of the various embedding EDS.

\section{The simplest case: a specialized embedding of minimal 2-spaces in flat 2+1-space}

With moving frame bases $\theta_{i}, i=1,2$, and $\theta_{A}, A=3$, signature $(1,1,-1)$ we begin with an EDS generated by $\theta_{3}$, its exterior derivative $\omega_{13} \wedge \theta_{1}+\omega_{23} \wedge \theta_{2}$, and the new specialization which in three dimensions is just the single form $\omega_{12}-\omega_{13}$. The character table is $6(2,1) 2$ with one degree of gauge freedom, well posed and involutory on $\theta_{1}$ and $\theta_{2}$. As RT theory does not exist in this low dimension we then consider extremal surfaces, with Lagrangian $\Lambda=\theta_{1} \wedge \theta_{2}$, whose variation generates the EDS to be the isometric embedding form $\theta_{3}$ together with the dynamic 2 -form $\omega_{13} \wedge \theta_{2}-\omega_{23} \wedge \theta_{1}$. This EDS for specialized minimal surfaces remains well posed, involutory on $\theta_{1}$ and $\theta_{2}$, with table $6(2,2) 2$.

Integration, introduction of scalar variables to explicitly find the PDE's, begins with the closed 1-form $\omega_{23}$, leading to a coordinate we call $u$. Sequentially from the structure (moving frame) equations and 2-forms in the EDS we find further closed 1-forms, and can introduce variables $v, x$ and $y$. The result is

$$
\omega_{23}=-u^{-1} d u, \quad \omega_{13}=u^{-1} d v, \quad \theta_{2}=u d x, \quad \theta_{1}=u d y,
$$

and the two 2 -forms require $u_{x}-v_{y}=u_{y}+v_{x}=0$. 
The induced intrinsic 2-metric on any of the family of solutions is then seen to be

$$
d s^{2}=\theta_{1} \theta_{1}+\theta_{2} \theta_{2}=u^{2}\left(d x^{2}+d y^{2}\right),
$$

where $u(x, y)$ satisfies Laplace's equation. The Gaussian curvature is $\left(u_{x}^{2}+u_{y}^{2}\right) / u^{4}$. We have not yet been able to characterize a common geometric property of this family of embedded minimal surfaces, resulting from various solutions of Laplace's equation; but it may be relevant that at least the plane, point particle and dipole solutions all lead to metrics that support a Killing vector field.

\section{Application to Einstein gravity}

Not surprisingly the symmetry specializations we have found to work with Regge-Teitelboim gravity of various classes appear to work also with Einstein gravity, but only in the class 6 case, embedding in flat 10-dimensional space with the required signature. Then indeed we find specialized frame bundles for setting EDS's for families of solutions of vacuum general relativity. This means we drop the strict isometric embedding 1-forms and return to the EDS (equally generated from the Hilbert Lagrangian) of four torsion 2-forms and four Ricci 3-forms.

Let us number bases $i=1,2,3,4$ and $A=5,6,7,8,9,0$, use signature $(-1,-1,-1,1,1,1,1$, $-1,1,1)$ and base our first specialization on indices 1 and 5 . Take the symmetry forms to be the c.i.p.s. $\left(\omega_{12}+\omega_{25}, \omega_{13}+\omega_{35}, \omega_{14}+\omega_{45}, \omega_{16}-\omega_{56}, \omega_{17}-\omega_{57}, \omega_{18}-\omega_{58}, \omega_{19}-\omega_{59}, \omega_{10}-\omega_{50}\right)$. The EDS is generated by these eight 1-forms, four torsion 2-forms $\omega_{i A} \wedge \theta_{A}$, their exterior derivatives, and the dynamic Ricci 3-forms $\omega_{i A} \wedge \omega_{A j} \wedge \theta_{k} \epsilon^{i j k l}$. Explicit calculation of the Cartan characters of this EDS shows it to be well posed and involutory on the $\theta_{i}: 55(8,4,12,14) 4$ with three degrees of gauge freedom. There are 10 Cauchy characteristic vectors $\left(\omega_{67}, \omega_{68}, \omega_{69}, \omega_{60}, \omega_{78}\right.$, $\omega_{79}, \omega_{70}, \omega_{89}, \omega_{80}, \omega_{90}$ do not appear in the generators of the EDS).

The second specialization adds the additional six symmetry forms based on indices 2 and 6 : $\left(\omega_{23}+\omega_{36}, \omega_{24}+\omega_{46}, \omega_{27}-\omega_{67}, \omega_{28}-\omega_{68}, \omega_{29}-\omega_{69}, \omega_{20}-\omega_{60}\right)$. Again well posed; $55(14,4,12,14) 4$, one degrees of gauge freedom and six CC vectors $\left(\omega_{78}, \omega_{79}, \omega_{70}, \omega_{89}, \omega_{80}, \omega_{90}\right.$ do not appear).

The third specialization adds four symmetry forms based on 3 and $7,55(18,4,12,14)$ with 2 CC's. The fourth specialization adds yet two more, based on 4 and 8, so 20 in all. This results in $55(20,4,12,14) 4$, with one remaining gauge freedom.

Acknowledgements. I thank the JPL Office of the Chief Scientist for a visiting appointment during which this research was carried out, and the Science Division for hospitality. My colleagues John W. Armstrong, Curt Cutler, Massimo Tinto and Michele Vallisneri gave constant stimulus and support.

\section{References}

[1] Estabrook F.B., Mathematical structure of tetrad equations for vacuum relativity, Phys. Rev. D 71 (2005), 044004, 5 pages, gr-qc/0411029.

[2] Estabrook F.B., The Hilbert Lagrangian and isometric embedding: tetrad formulation of Regge-Teitelboim gravity, J. Math. Phys. 51 (2010), 042502, 10 pages, arXiv:0908.0365.

[3] Estabrook F.B., Wahlquist H.D., Moving frame formulations of 4-geometries having isometries, Classical Quantum Gravity 13 (1996), 1333-1338.

[4] Ivey T.A., Landsberg J.M., Cartan for beginners: differential geometry via moving frames and exterior differential systems, Graduate Studies in Mathematics, Vol. 61, Amer. Math. Soc., Providence, RI, 2003.

[5] Paston S.A., Sheykin A.A., Embeddings for the Schwarzschild metric: classification and new results, Classical Quantum Gravity 29 (2012), 095022, 17 pages, arXiv:1202.1204.

[6] Stephani H., Kramer D., MacCallum M., Hoenselaers C., Herlt E., Exact solutions of Einstein's field equations, 2nd ed., Cambridge Monographs on Mathematical Physics, Cambridge University Press, Cambridge, 2003. 